\title{
Multipotent mesenchymal stromal cell-derived exosomes improve functional recovery after experimental intracerebral hemorrhage in the rat
}

\author{
Yuxia Han, BS, ${ }^{1}$ Don Seyfried, BS, ${ }^{2}$ Yuling Meng, PhD, ${ }^{1}$ Dongmei Yang, MD, ${ }^{1}$ Lonni Schultz, PhD, ${ }^{3}$ \\ Michael Chopp, PhD, ${ }^{2}$ and Donald Seyfried, MD' \\ Departments of ${ }^{1}$ Neurosurgery, ${ }^{2}$ Neurology, and ${ }^{3}$ Public Health Sciences, Henry Ford Hospital, Detroit, Michigan
}

\begin{abstract}
OBJECTIVE Previous studies have demonstrated that transplanted multipotent mesenchymal stromal cells (MSCs) improve functional recovery in rats after experimental intracerebral hemorrhage $(\mathrm{ICH})$. In this study the authors tested the hypothesis that administration of multipotent MSC-derived exosomes promotes functional recovery, neurovascular remodeling, and neurogenesis in a rat model of $\mathrm{ICH}$.
\end{abstract}

METHODS Sixteen adult male Wistar rats were subjected to $\mathrm{ICH}$ via blood injection into the striatum, followed 24 hours later by tail vein injection of $100 \mu \mathrm{g}$ protein of MSC-derived exosomes (treatment group, 8 rats) or an equal volume of vehicle (control group, 8 rats); an additional 8 rats that had identical surgery without blood infusion were used as a sham group. The modified Morris water maze (mMWM), modified Neurological Severity Score (mNSS), and social odor-based novelty recognition tests were performed to evaluate cognitive and sensorimotor functional recovery after ICH. All 24 animals were killed 28 days after ICH or sham procedure. Histopathological and immunohistochemical analyses were performed for measurements of lesion volume and neurovascular and white matter remodeling.

RESULTS Compared with the saline-treated controls, exosome-treated ICH rats showed significant improvement in the neurological function of spatial learning and motor recovery measured at $26-28$ days by mMWM and starting at day 14 by mNSS $(p<0.05)$. Senorimotor functional improvement was measured by a social odor-based novelty recognition test $(p<0.05)$. Exosome treatment significantly increased newly generated endothelial cells in the hemorrhagic boundary zone, neuroblasts and mature neurons in the subventricular zone, and myelin in the striatum without altering the lesion volume.

CONCLUSIONS MSC-derived exosomes effectively improve functional recovery after $\mathrm{ICH}$, possibly by promoting endogenous angiogenesis and neurogenesis in rats after ICH. Thus, cell-free, MSC-derived exosomes may be a novel therapy for $\mathrm{ICH}$.

https://thejns.org/doi/abs/10.3171/2018.2.JNS171475

KEYWORDS exosomes; experimental intracerebral hemorrhage; functional recovery; mesenchymal stromal cell; angiogenesis; neurogenesis

I NTRACEREBRAL hemorrhage ( $\mathrm{ICH})$ is a devastating form of stroke with high rates of mortality and morbidity. ${ }^{15}$ In patients surviving the initial expansion of the clot, the brain is susceptible to secondary injury from inflammation and the coagulation cascade in the perihematomal regions. ${ }^{14,58}$ Research efforts have focused on pharmacological enhancement of neuroprotection from the acute injury. ${ }^{16,46}$ Alternatively, therapies can be designed to augment the natural recovery process that occurs in the brain as patients regain function, termed the neurorestorative approach. Using this approach, the transplantation of bone marrow mesenchymal stromal cells (MSCs) has been extensively studied as a treatment for stroke. MSCs home to damaged microvasculature based on matching pairs of membrane adhesion proteins and have proven effective at enhancing functional recovery following experimental

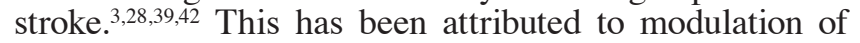
immune and endothelial cells, as well as neurons and glia,

ABBREVIATIONS ANOVA = analysis of variance; $\mathrm{BBB}=$ blood-brain barrier; $\mathrm{ICH}=$ intracerebral hemorrhage; $\mathrm{mMWM}=$ modified $\mathrm{Morris}$ water maze; $\mathrm{mNSS}=$ modified Neurological Severity Score; MSC = mesenchymal stromal cell; OPC = oligodendrocyte progenitor cell; PBS = phosphate-buffered saline; SE = standard error; SVZ = subventricular zone; $\mathrm{TBI}=$ traumatic brain injury. SUBMITTED June 19, 2017. ACCEPTED February 16, 2018.

INCLUDE WHEN CITING Published online July 20, 2018; DOI: 10.3171/2018.2.JNS171475. 
via cytokines or factors secreted by MSCs. ${ }^{9,17}$ Emerging data show that one way MSCs affect recovery is by secretion of exosomes, which are extracellular vesicles composed of protein, lipids, mRNA, and miRNA.?

Exosomes are formed from the inward budding of a large intracellular vesicle called a multivesicular body, trapping cytoplasm components and cargo proteins in a smaller compartment $(30-120 \mathrm{~nm}) .^{19}$ When the multivesicular body merges with the plasma membrane, the exosomes are released into the interstitial fluid but their contents are protected from extracellular RNAase and other digestive enzymes. This process makes exosomes effective vehicles in their ability to transfer RNA to distant cells.

Recent studies on multipotent MSC-derived exosomes have demonstrated their effectiveness in treating cardiovascular, liver, and kidney diseases. ${ }^{7,17}$ Our previous studies demonstrated that MSC-derived exosomes promote neurite remodeling and functional recovery in rats after ischemic stroke and traumatic brain injury (TBI). ${ }^{49,50,55}$ The present study aimed to investigate the potential therapeutic benefit of intravenously administered exosomes in a rat model of experimental ICH by posttreatment measurement of cognitive and sensorimotor functional recovery as well as exploring the potential underlying cellular mechanisms.

\section{Methods}

All experimental procedures were approved by the Henry Ford Health System Institutional Animal Care and Use Committee. An ICH injection model in adult male Wistar rats (weighing 270-300 g, from Charles River) was used. ${ }^{39}$ Briefly, blood was taken from the femoral artery and after craniectomy, injected into the striatum 3-4 mm lateral to bregma and 5-mm deep into the brain; $0.1 \mathrm{ml}$ of blood was infused at a rate of $10 \mu \mathrm{l}$ per minute to create the hemorrhage. The animals were randomly divided into 3 groups (8 rats per group): group 1 received exosomes derived from $3 \times 10^{6} \mathrm{MSCs}$ (in $0.5 \mathrm{ml}$ phosphate-buffered saline [PBS]); group 2 was treated with equal volume (0.5 $\mathrm{ml}$ ) of PBS; and group 3 (sham group) had surgery without blood infusion into the brain and was treated with 0.5 $\mathrm{ml}$ PBS. The exosome solution or PBS was administered intravenously over 5 minutes via the tail vein, starting 24 hours after the ICH; this protocol was chosen based on our ischemic stroke and TBI studies with exosomes in rats. ${ }^{55}$ All rats were killed at 28 days after treatment. In addition, all rats received a daily intraperitoneal injection of 100 $\mathrm{mg} / \mathrm{kg}$ 5'-bromo-2'-deoxyuridine (Brdu; Sigma) from day 1 until day 14.

\section{Neurological Functional Studies}

A modified Morris water maze (mMWM) test was used to monitor cognitive (spatial learning) improvement. The spatial learning testing with $\mathrm{mMWM}$ was identical to that previously described., ${ }^{1,2}$ The mMWM testing was performed from day 24 through day 28 after experimental ICH. Data collection was automated by the HVS Image 2020 Plus tracking system. Each rat was given 4 trials per day for 4 days with a hidden platform at a fixed location, and then 4 trials were performed on day 5 with the plat- form in different locations hidden beneath the surface of the water. Each trial lasted a maximum of 90 seconds. If the animal reached the platform within 90 seconds, the percentage of time traveled within the northeast quadrant (where the hidden platform was located) was calculated relative to the total amount of time spent swimming before reaching the platform, and this percentage was employed for statistical analysis.

The modified Neurological Severity Score (mNSS) is used to assess neurological function via a $0-18$ composite score of motor, sensory, balance, and reflex measures, with higher scores implying greater neurological injury. ${ }^{41,42}$

Odor recognition was used to test sensory memory ability in identifying a previously encountered stimulus. The odor recognition test was performed in the home cage as previously described in detail ${ }^{35}$ and consisted of 3 phases: a familiarization phase, an odor habituation phase, and an odor recognition memory test phase. The memory test phase gives the rat a choice of 4 beads: 3 beads (F1-F3) that are saturated with the familiar smell of the home cage and 1 bead (N1) with the smell of a separately housed rat. Three trials are run for 1 minute, switching the place of the beads for each trial. The amount of time spent sniffing each bead is noted. The next day, a new bead with the smell of an unfamiliar rat (N2) is presented alongside F1, $\mathrm{F} 2$, and N1, and 3 more trials are run. The more time the rat smells the $\mathrm{N} 2$ bead compared to the $\mathrm{N} 1$ bead represents how well the rat has remembered the N1 smell from the day before.

\section{Exosome Generation and Preparation From MSC Cultures}

MSC expansion was performed according to previously described methods.$^{50}$ Bone marrow from adult male Wistar rats was mechanically harvested by flushing the cavity of the femurs with PBS, and the cells were washed and suspended in culture medium..$^{50}$ The initial cultured cells were considered to be P0 MSCs. The MSCs were conventionally cultured with a modified minimum essential medium (Hyclone) containing 20\% fetal bovine serum (FBS) and penicillin-streptomycin. For the exosome isolation, exosome-depleted FBS was used (EXO-FBS-250 A-1, System Biosciences). When the cells reached $60 \%$ to $80 \%$ confluence, they were cultured for an additional 48 hours. The media were collected and exosomes were isolated using the ExoQuick exosome isolation method according to the manufacturer's instructions (System Biosciences). Next, $2.5 \mathrm{ml}$ of ExoQuick-TC was added to $10 \mathrm{ml}$ of media, and the mixture was incubated for 12 hours at $4^{\circ} \mathrm{C}$, then centrifuged at $1500 \mathrm{~g}$ for 30 minutes. After the supernatant (nonexosomal fraction) was removed from the samples, the exosome pellets were washed 3 times with PBS to remove chemical residue and then suspended in $200 \mu \mathrm{l}$ of PBS. The total protein content of the exosome concentration was quantitated using bicinchoninic acid (BCA) assay (Pierce), and particle size was analyzed using a qNano nanopore-based exosome detection system according to the manufacturer's instructions (Izon). Exosomes were identified by a specific protein marker Alix by Western blot, and electron microscopy was performed to verify their presence, as described in our previous study. ${ }^{48}$ 


\section{Histology and Immunohistochemistry}

After the behavioral tests at the 28-day time point, all animals from all 3 groups were anesthetized and perfused transcardially with PBS, followed by $4 \%$ paraformaldehyde in PBS. Brain tissues were excised, fixed in $4 \%$ paraformaldehyde, and sliced into seven 2-mm-thick blocks. Each block was processed and embedded in paraffin, and 4 adjacent 6 - $\mu$ m-thick sections were cut from each block and stained with hematoxylin and eosin ( $\mathrm{H} \& \mathrm{E})$. The percentage of tissue loss in the ipsilateral hemisphere compared to the contralateral hemisphere of the brain was measured using an image analysis system (Data Translation), as previously described..$^{39}$

For immunohistological studies, $6-\mu \mathrm{m}$-thick sections were blocked in a Tris-buffered saline solution containing $5 \%$ normal goat serum, $1 \%$ BSA, and $0.05 \%$ Tween-20. Sections were then incubated with the primary antibodies for localization of $\mathrm{BrdU}$ (1:100; a marker for proliferation cells, monoclonal antibody against BrdU, Roche), EBA (1:1000; a marker for detection of mature vessels, endothelial barrier antigen, Covance), TUJ1 (1:400; monoclonal antibody against tubulin isotype III, BABCO), DCX (1:50; Doublecortin, a marker for migrating neuronal precursor cells, Santa Cruz Biotechnology), and MAP2 (1:1000; a marker for neuron-specific cytoskeletal proteins, Abcam). The sections were incubated with Cy3- and/or FITCconjugated secondary antibody (1:200; Jackson ImmunoResearch) at room temperature for 2 hours. Nuclei were counterstained with DAPI (4',6-diamidino-2-phenylindole, Sigma).

White matter was stained using $0.1 \%$ Luxol fast blue (Poly Scientific R\&D). Sections were dehydrated with 95\% alcohol and stained in Luxol fast blue overnight. Excess stain was removed with $95 \%$ alcohol, and the sections were rinsed in distilled water. Immersion in $0.05 \%$ lithium carbonate solution differentiated between gray and white matter. Sections were again dehydrated with $95 \%$ alcohol, cleared in xylene, and then mounted in xylene-soluble mounting media.

Quantitative measurements of immunostaining were performed by an observer blinded to the treatment status of the individual animals. All slides were digitized under a $\times 20$ objective lens (Eclipse $80 i$, Nikon) by using a CoolSNAP color camera (Photometrics) interfaced with a MetaMorph image analysis system (Molecular Device). BrdU-positive cells in the hemorrhagic boundary zone and subventricular zone (SVZ) were digitized and counted and the counts from multiple fields of view were converted to units of cell number $/ \mathrm{mm}^{2}$. The same process was applied to quantification of EBA- and Map2-positive cells. TUJ1 and DCX were quantified as percentage of positive area (above threshold) per field of view. For quantification of vessels, the number of EBA-positive vessels was measured in 8 fields of view within the hemorrhagic boundary zone and SVZ. Data are presented as the density of EBAimmunoreactive vessels relative to the area $\left(\right.$ vessels $/ \mathrm{mm}^{2}$ ).

\section{Statistical Analysis}

Analysis of variance (ANOVA) with repeated-measures analyses was done to assess the group effects over time (or conditions for odor test) for the neurological functional
TABLE 1. Comparison of mMWM scores in the sham, control, and exosome-treated groups

\begin{tabular}{cccc}
\hline Time & Sham & Control & Exosome-Treated \\
\hline Day 24 & $39.07 \pm 1.94$ & $33.72 \pm 4.46$ & $37.49 \pm 3.31$ \\
\hline Day 25 & $44.07 \pm 2.92$ & $36.27 \pm 3.34$ & $42.67 \pm 4.97$ \\
\hline Day 26 & $52.82 \pm 2.23$ & $38.92 \pm 2.90$ & $47.64 \pm 2.53$ \\
\hline Day 27 & $64.07 \pm 2.92$ & $41.05 \pm 3.06$ & $49.36 \pm 2.38$ \\
\hline Day 28 & $54.07 \pm 2.92$ & $38.83 \pm 3.14$ & $46.69 \pm 1.55$ \\
\hline
\end{tabular}

Data represent mean $\pm \mathrm{SE}$.

scores. If the interaction between time and group was significant $(\mathrm{p}<0.05)$, pairwise comparisons of the groups were done within each time point using ANOVA methods. For pairwise comparisons of time points or conditions within a group, paired t-tests were done. ANOVA methods were also used for immunohistochemical outcomes with similar pairwise comparisons of the groups when the overall difference was significant. The testing level was set at 0.05 , with no adjustments for multiple comparisons in this proof-of-concept study. Data are presented as the mean \pm standard error (SE). All measurements were performed by observers blinded to individual treatments.

\section{Results \\ MSC Exosome Administration Significantly Enhances Spatial Learning in Rats After ICH}

Spatial learning measurements were performed during the last 5 days (24-28 days after ICH) using an mMWM test. The greater the percentage of time the animals spent in the correct quadrant (i.e., the northeast quadrant) of the water maze, the better the spatial learning. Over time the 3 groups followed different trends $(\mathrm{p}=0.034$ for interaction between group and time). In the testing of spatial learning, no significant between-group differences in the time spent in the correct quadrant were detected on the 1st and 2nd days of testing (i.e., the 24th and 25th days after ICH). The percentage of time spent by sham rats in the correct quadrant increased significantly from 26 to 28 days after sham operation, compared with time spent in the correct quadrant on the first 2 days of testing (days 24 and 25) (p $<0.003$ for all). The time spent in the correct quadrant (northeast) by all ICH rats gradually increased from day 24 to day 28 after surgery, but the increases were not statistically significant. Compared to the control group, the exosome-treated group showed significant improvement in spatial learning (that is, a larger percentage of time spent in the correct quadrant) at days 26, 27, and 28 after $\mathrm{ICH}(\mathrm{p}=0.025, \mathrm{p}=0.048$, and $\mathrm{p}=0.046$, respectively; Table 1 and Fig. 1).

\section{MSC Exosome Administration Significantly Improves Neurological Functional Recovery in Rats After ICH}

The mNSS was performed to test motor coordination and sensory ability. Foot faults during grid walking, direction bias when the rat is held by the tail, and a slow reaction time to remove a sticker from the paw are all indicative of neurological damage. As in the mMWM scores, 


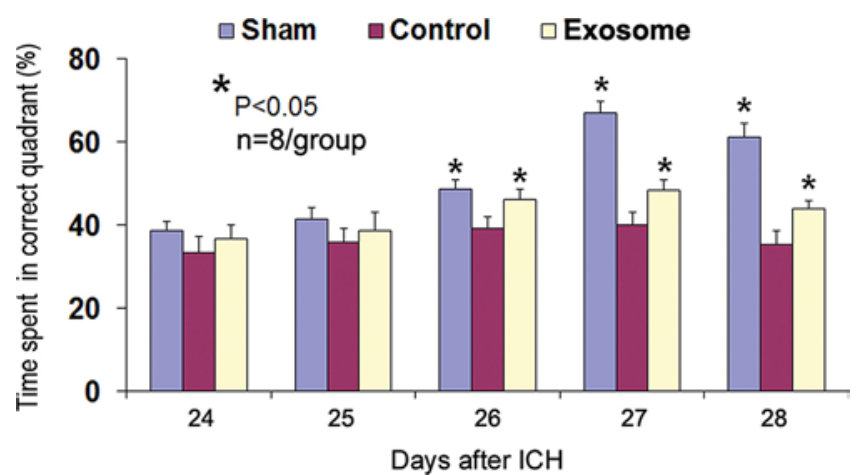

FIG. 1. Exosome treatment significantly improves the spatial learning function measured by mMWM test from day 26 to day 28 after ICH in rats. Data represent mean \pm SE (error bars); $n=8$ per group. Figure is available in color online only.

the 3 groups showed different trends over time $(\mathrm{p}<0.001$ for interaction between group and time). Compared to rats in the sham group, ICH rats in the control and exosometreatment groups showed severe neurological impairment in the mNSS test on day 1 ( $\mathrm{p}<0.001$ for both). At 1 week after $\mathrm{ICH}$, there was no significant difference between the exosome-treated group and the control group in terms of mNSS results $(p=0.746)$, but starting at day 14 , the exosome-treated group showed significantly better improvement in neurological function than the control group (day $14, \mathrm{p}=0.016$; day $21, \mathrm{p}=0.007$; and day $28, \mathrm{p}<0.001$; Table 2 and Fig. 2).

\section{Exosome Treatment Improves Nonspatial Social Odor-Based Recognition}

The social odor-based recognition task was performed to detect sensory-memory deficits after ICH that might be related to anterograde amnesia. The 3 groups again followed different trends over the 4 different conditions or beads ( $p<0.001$ for interaction between group and condition). The mean amount of time spent with the familiarsmell beads was similar across all groups $(\mathrm{F} 1, \mathrm{p}=0.936$; and F2, p > 0.99). Also, the mean amount of time spent on the odor bead from the day before (N1) was similar across all groups $(p=0.412)$. However, the rats in the control group spent significantly less time exploring the novel odor bead (N2) than did those in the sham and exosometreated groups $(\mathrm{p}<0.01$ and $\mathrm{p}=0.001$, respectively) (Table 3 and Fig. 3). These data indicate that nonspatial social

TABLE 2. Comparison of mNSS scores in the sham, control, and exosome-treated groups

\begin{tabular}{cccc}
\hline Time & Sham & Control & Exosome-Treated \\
\hline Day 1 & $2.38 \pm 0.42$ & $9.75 \pm 0.16$ & $9.88 \pm 0.12$ \\
\hline Day 7 & $0.63 \pm 0.33$ & $7.88 \pm 0.40$ & $7.13 \pm 0.52$ \\
\hline Day 14 & $0.13 \pm 0.12$ & $6.00 \pm 0.33$ & $4.88 \pm 0.40$ \\
\hline Day 21 & $0.00 \pm 0.00$ & $5.38 \pm 0.33$ & $4.38 \pm 0.26$ \\
\hline Day 28 & $0.00 \pm 0.00$ & $4.38 \pm 0.33$ & $3.13 \pm 0.23$ \\
\hline
\end{tabular}

Data represent mean $\pm \mathrm{SE}$.

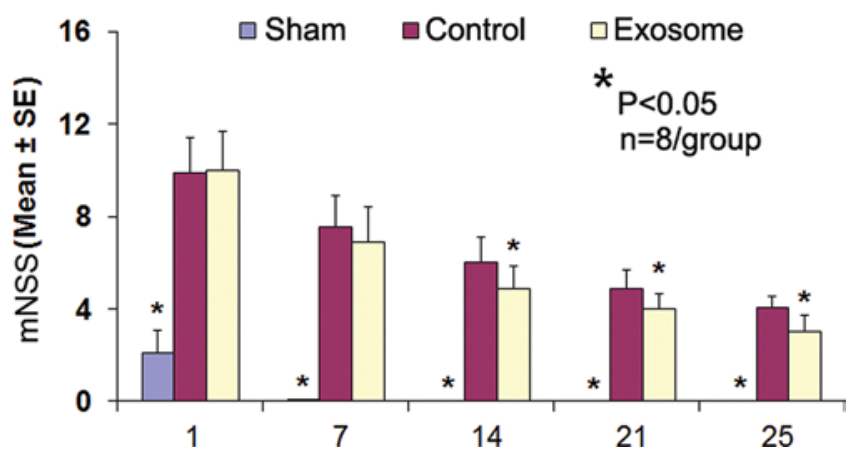

FIG. 2. Treatment of ICH in rats with MSC-derived exosomes significantly improves sensorimotor function measured by mNSS starting at day 14 and continuing through day 25 after $\mathrm{ICH}$. Data represent mean \pm $\mathrm{SE}$ (error bars); $\mathrm{n}=8$ per group. Figure is available in color online only.

odor-based memory impairment occurred after ICH and that exosome treatment starting 24 hours after ICH enhanced recovery of sensory memory.

\section{MSC Exosome Administration Significantly Increases Vascular Density and Angiogenesis in Rats After ICH}

To quantitate changes in vessel density after ICH, EBA staining was performed. The overall difference among the 3 groups with respect to the density of EBA-positive vessels was statistically significant $(\mathrm{p}=0.007)$. Compared to the control group, the exosome-treated group demonstrated significantly increased density of EBA-positive vessels in the hemorrhagic boundary zone ( $389.95 \pm 47.30$ vessels/ $\mathrm{mm}^{2}$ for control vs $579.46 \pm 55.82$ vessels $/ \mathrm{mm}^{2}$ for exosome-treated, $\mathrm{p}=0.008)$, as did the sham group (598.70 \pm 31.31 vessels $/ \mathrm{mm}^{2}, \mathrm{p}=0.007$ vs control). The difference between the exosome-treated and sham groups was not statistically significant $(\mathrm{p}=0.77)(\mathrm{Fig} .4)$.

\section{Administration of MSC-Derived Exosomes Significantly Increases Neurogenesis in the Subventricular Zone in Rats After ICH}

To determine the effects of exosomes on cell proliferation after ICH, we injected BrdU into rats once daily for 14 days starting 24 hours after ICH. Animals were killed 28 days after ICH, and immunostaining was performed on paraffin-embedded brain coronal sections. ${ }^{40-42}$ The overall difference among the 3 groups for BrdU was significant $(p<0.001)$. Exosome treatment increased the amount of BrdU-positive cells in the SVZ compared to the control group, with the exosome-treated group and sham group

TABLE 3. Comparison of odor test results in the sham, control, and exosome-treated groups

\begin{tabular}{cccc}
\hline Bead & Sham & Control & Exosome-Treated \\
\hline F1 & $0.88 \pm 0.29$ & $0.88 \pm 0.29$ & $0.75 \pm 0.25$ \\
\hline F2 & $0.75 \pm 0.25$ & $0.75 \pm 0.31$ & $0.75 \pm 0.25$ \\
\hline N1 & $2.00 \pm 0.33$ & $1.38 \pm 0.37$ & $1.50 \pm 0.33$ \\
\hline N2 & $13.75 \pm 2.21$ & $3.50 \pm 0.50$ & $12.63 \pm 1.89$ \\
\hline
\end{tabular}

Data represent mean time in seconds $\pm \mathrm{SE}$. 


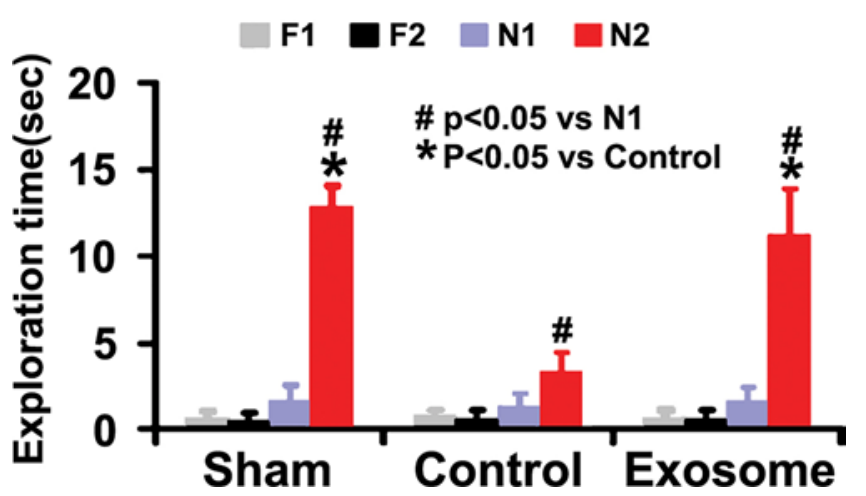

FIG. 3. Exosome treatment significantly improves nonspatial social odor-based novelty recognition memory at day 28 after $\mathrm{ICH}$ in rats. The exosome-treated rats explored the novel odor bead (N2) significantly more than the control rats. Data represent mean \pm SE (error bars); $n=8$ per group. Figure is available in color online only.

having similar numbers of BrdU-positive cells per $\mathrm{mm}^{2}$ (Fig. 5; $988.24 \pm 73.34$ for the sham group, $542.84 \pm 64.83$ for the control group, and $926.31 \pm 69.79$ for the exosometreated group; $\mathrm{p}<0.001$ for control vs the sham and exosome-treated groups and $\mathrm{p}=0.534$ for sham vs exosometreated groups).

To determine the effects of exosomes on neurogenesis, immunostaining for DCX, TUJ1, and MAP2 was performed (Figs. 5 and 6). Treatment with exosomes significantly increased DCX and MAP2 expression compared with the control ICH group $(12.06 \% \pm 1.49 \%$ vs $17.56 \%$ $\pm 1.67 \%$ for DCX, $\mathrm{p}=0.04$; and $385.22 \% \pm 49.39 \%$ vs $541.33 \% \pm 54.58 \%$ for MAP2, $\mathrm{p}=0.045)$ and showed a strong trend for TUJ1 $(11.93 \% \pm 1.53 \%$ vs $17.30 \% \pm$ $1.81 \%, \mathrm{p}=0.053$ ). Results in the sham group were also significantly higher than in the control group for all 3 measures (DCX: $18.81 \% \pm 21.4 \%, \mathrm{p}=0.014$; MAP2: $567.64 \% \pm 51.76 \%, \mathrm{p}=0.021$; and TUJ1: $18.68 \% \pm 2.17 \%$, $\mathrm{p}=0.017)$ and similar to results in the exosome-treated group (DCX, $\mathrm{p}=0.628$; MAP2, $\mathrm{p}=0.723$; and TUJ1, $\mathrm{p}=$ 0.605). Double staining for BrdU-DCX, BrdU-TUJ1, and BrdU-MAP2 (Figs. 5 and 6) revealed that some neuronal marker-positive cells were BrdU-positive, indicating that there were newly generated neuroblasts (BrdU-DCX, BrdU-TUJ1) and newly generated immature neurons (BrdU-MAP2) around the hematoma and the SVZ. We found that exosome treatment significantly increased the number of DCX/BrdU+ cells $(9.74 \pm 1.23$ vs $5.61 \pm 1.23$, $\mathrm{p}=0.028), \mathrm{TUJ} 1 / \mathrm{BrdU}+$ cells $(9.77 \pm 0.99$ vs $5.42 \pm 0.82$, $\mathrm{p}=0.004)$, and $\mathrm{MAP} 2 / \mathrm{BrdU}+$ cells $(42.13 \pm 7.37$ vs 19.20 $\pm 2.30, \mathrm{p}=0.01)$ compared to the results in the ICH control group.

\section{Exosome Treatment Increases White Matter Density in the Striatum}

Myelin was stained using Luxol fast blue. The myelinpositive area in the perihematomal region was quantified through thresholding and then divided by the myelin-positive area in the contralateral hemisphere to generate the myelin fraction (Fig. 7). In the striatum, exosome treatment improved the myelin fraction compared to results in controls $(0.86 \pm 0.047$ vs $0.71 \pm 0.043$, p < 0.05$)$, but did not completely recover myelin to the levels found in the sham group $(0.96 \pm 0.038, p<0.05)$. In the corpus callosum, exosome treatment did not significantly increase myelin fraction compared to results in the control group $(0.86 \pm 0.051$ vs $0.73 \pm 0.059, \mathrm{p}=0.12)$.

\section{Effects of Exosome on Tissue Loss After ICH}

Measurements of tissue volume in the injured hemisphere showed significant loss in the saline-treated and exosome-treated groups compared to the sham group at 28 days after ICH (Fig. 8). Tissue loss estimates for the saline- and exosome-treated groups were not significantly different from each other $(28.90 \% \pm 3.25 \%$ for control and $27.04 \% \pm 3.20 \%$ for exosome-treated, $\mathrm{p}=0.622$ ) and both were significantly higher than tissue loss estimates for the sham group $(1.01 \% \pm 0.16 \%, \mathrm{p}<0.001)$.

\section{Discussion}

In the present study, we demonstrate that systemic administration of cell-free MSC-derived exosomes after ICH in rats does not prevent tissue loss. However, this treatment showed the following positive effects: 1) improved spatial learning (mMWM test), motor function (mNSS test), and sensory memory (odor recognition test); 2) increased vessel density in the hematoma boundary zone; 3 ) increased numbers of newborn neuroblasts and mature neurons in the SVZ; and 4) increased myelin staining in the striatum. Our results suggest that improved functional recovery after exosome treatment in this model may be associated with increased angiogenesis and neurogenesis. The administration of MSC-derived exosomes may rep-
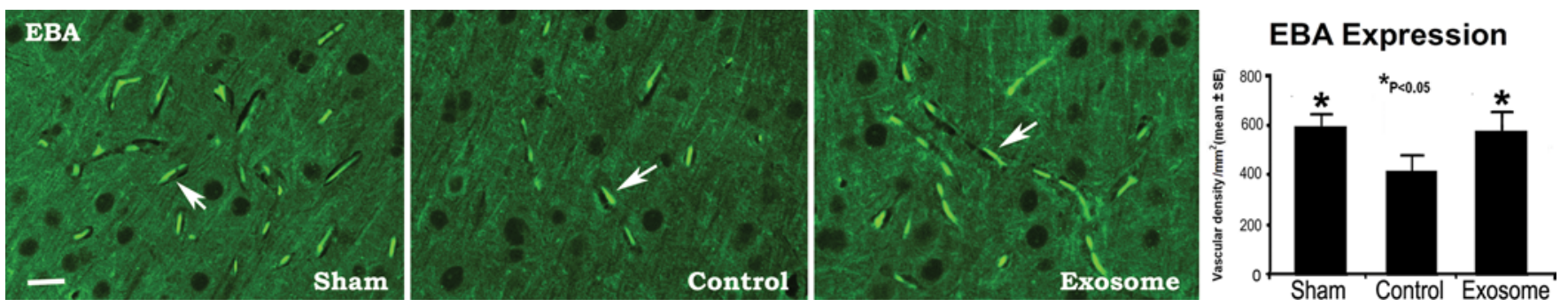

FIG. 4. Exosome treatment significantly increases vascular density in the brain after ICH in rats. EBA staining was performed for detection of mature vasculature at day 28 after $\mathrm{ICH}$ in the lesion boundary zone. Scale bar $=25 \mu \mathrm{m}$. Data in bar graph (right panel) represent mean $\pm \mathrm{SE}$ (error bars); $\mathrm{n}=8$ per group. 

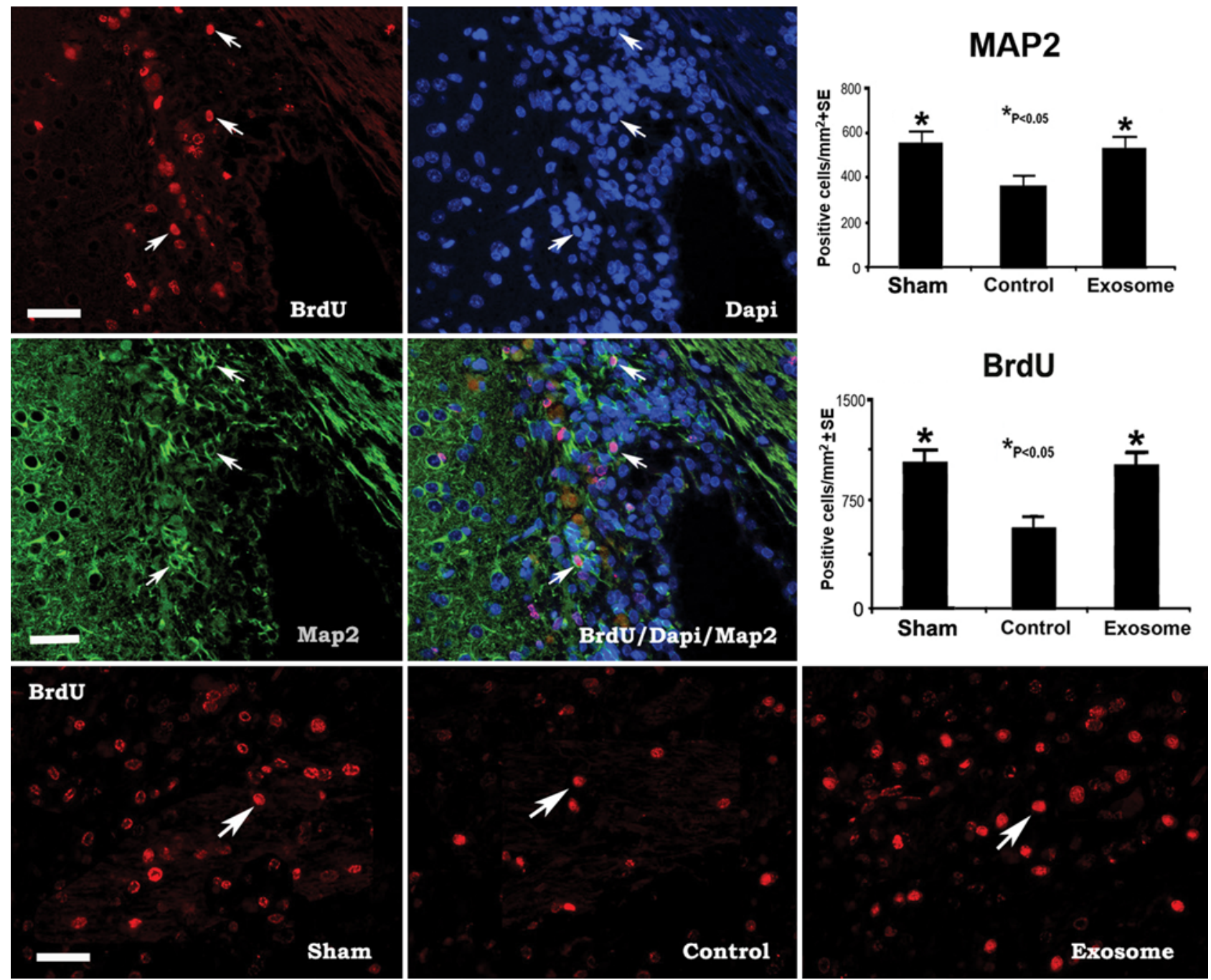

FIG. 5. Exosome treatment significantly increases neurogenesis in the SVZ of rats killed at day 28 after ICH. BrdU staining highlights cell proliferation. MAP2 staining identifies mature neurons, and BrdU (red)/MAP2 (green) indicates newborn mature neurons. Dapi identifies cell nuclei. BrdU and MAP2 colocalize within newly formed mature neurons. The top 4 photographs (first and second rows) show the appearance of BrdU, Dapi, and MAP2 and the colocalization of staining in a specimen from an exosome-treated rat. The bottom 3 fluorescent images show BrdU staining in sections from a sham, a control, and an exosometreated rat. Scale bar $=25 \mu \mathrm{m}$. Data in bar graphs represent mean $\pm \mathrm{SE}$ (error bars); $\mathrm{n}=8$ per group.

resent a novel therapeutic approach warranting further investigation for treatment of ICH.

ICH triggers a cascade of changes to the brain that destroys neural structure and creates mass effect and subsequent perihematomal edema. In the border zone of edema, cells are vulnerable to damage by ectopic serum proteins (thrombin) and hemoglobin breakdown products. An inflammatory reaction recruits immune cells, which help phagocytize much of this ectopic material but also may damage surrounding brain cells with their release of cytokines. ${ }^{29}$ The border zone may undergo extensive remodeling to compensate for loss of neuronal function in the core ${ }^{34}$ It is during the remodeling process that proliferating vessels, neuronal precursors, and glial cells likely play a key role in structural and functional recovery. ${ }^{6}$ That treatment with exosomes may modulate the inflammatory process and influence recovery after ICH remains a pos- sibility that was not addressed in our study but should be investigated in the future.

In this 28-day study, the exosome treatment significantly improved functional recovery (mMWM, mNSS, odor test) after ICH compared to results in the control animals. The comprehensive mNSS provides multiple measures of sensory-motor coordination, including limb placement, grip, and balance. It demonstrated that both control and exosome-treated groups had profound motor deficits immediately after the ICH, but at day 14 the recovery of the exosome-treated rats became significantly greater than that of controls. The deficits in the ICH rats and the improvements seen in the exosome group with the odor test, despite the injury site being distal to the olfactory bulb, suggest that the hematomal region is important for the functional integration of olfaction and social memory. 

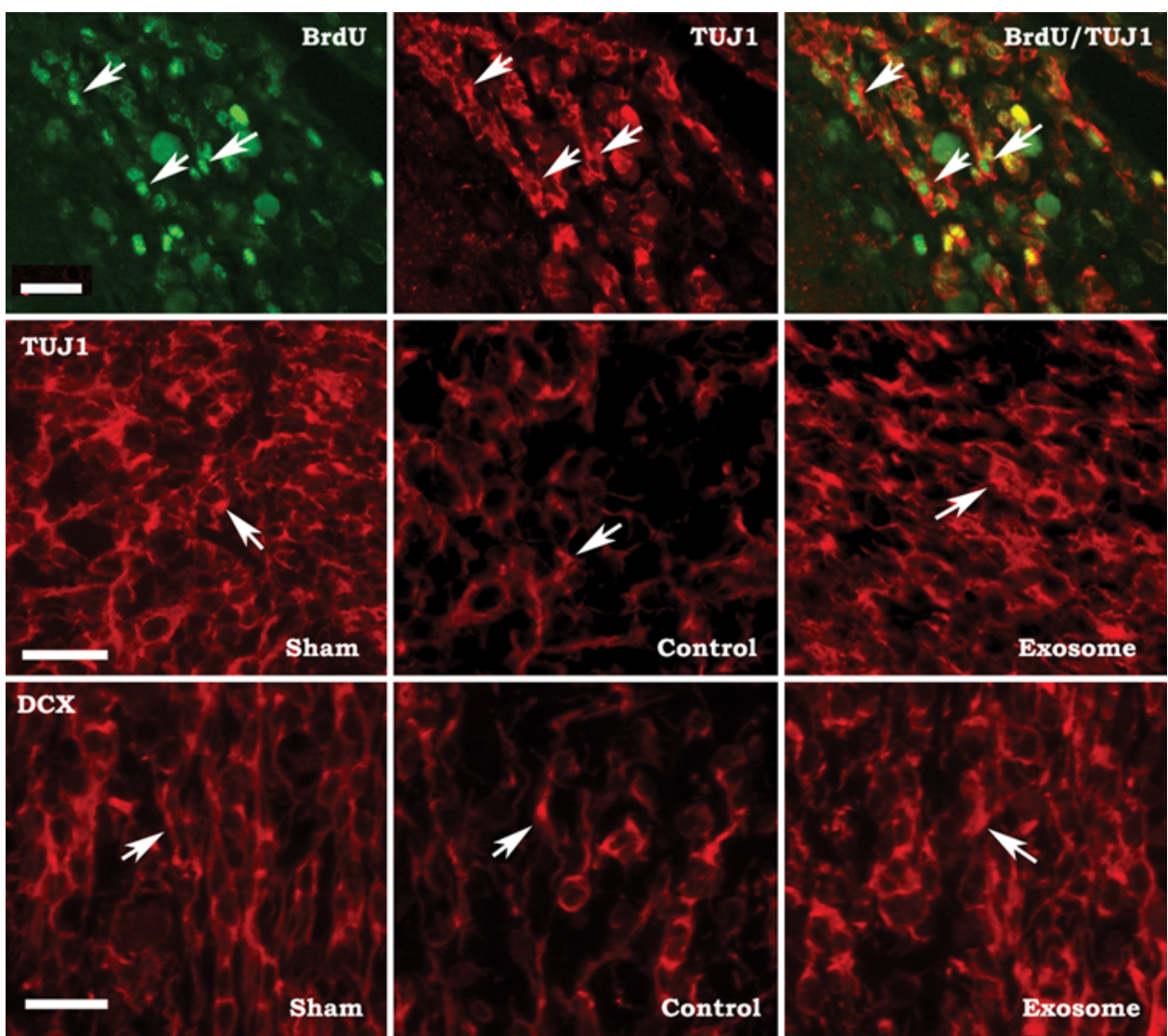

TUJ1
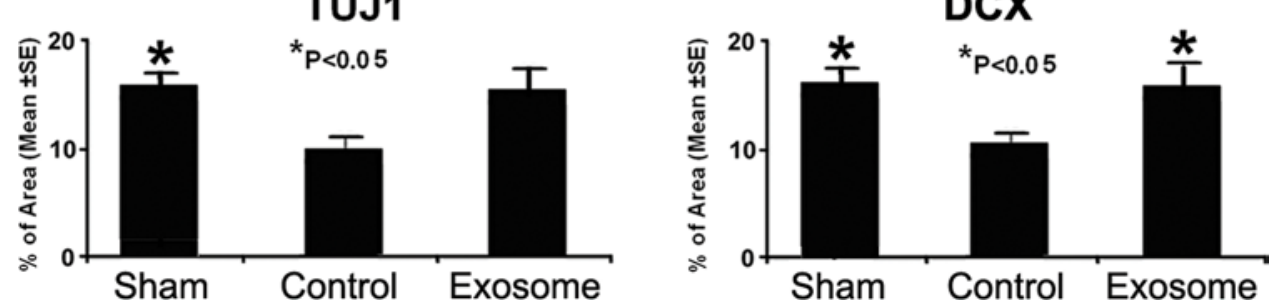

FIG. 6. Exosome treatment significantly increases cell proliferation in the SVZ at day 28 after ICH. BrdU staining was performed for cell proliferation, and DCX and TUJ1 staining was performed for immature neurons. The top row shows double staining with BrdU (green)/TUJ1 (red) for newborn immature neurons in a specimen from an exosome-treated rat. The lower 2 rows of photographs demonstrate the difference between DCX and TUJ1 in the sham, control, and exosome groups. The bottom graphs show the statistical comparison of quantified staining across the 3 groups. Of note, the TUJ1 comparison resulted in a near-significant increase in the exosome-treated group relative to the control group $(p=0.05)$. Scale bar $=25 \mu \mathrm{m}$. Data in bar graphs represent mean $\pm \mathrm{SE}$ (error bars); $\mathrm{n}=8$ per group.

Exosome treatment following ICH, compared to control, increased the density of EBA-positive cells. The increased density of these cells indicates mature vessels with a functioning blood-brain barrier (BBB) ${ }^{38}$ It is probable that the higher vessel density is due to angiogenesis that produced mature vessels in the course of the 1-month recovery time. Angiogenesis can contribute to functional recovery by accelerating neurite growth and synaptogenesis in the brain. This is similar to the effect of exosomes on recovery from ischemic stroke. ${ }^{7}$ Clinically, ischemia after hemorrhage is uncommon, but hyperemia in injured brain regions compared to the contralateral hemisphere is associated with better functional outcome, implicating increased angiogenesis as crucial to recovery. ${ }^{5} \mathrm{VEGF}$, an angiogenic hormone, acts through endothelial nitric oxide synthase-BDNF (brain-derived neurotrophic factor) pathway to promote neurogenesis after stroke. ${ }^{4}$ Angiogenesis is well coupled with neurogenesis, sharing factors that are essential for dividing cells and simultaneously building a delivery system for long-term metabolic support of new neurons. The newly formed vasculature also provides the substrate for new neuron migration after injury to the adult striatum, ${ }^{22}$ diverting neurons from the canonical rostral migratory stream to the olfactory bulb. ${ }^{21} \mathrm{SVZ}$ explants in coculture with endothelial cells have been shown to increase neuronal maturation..$^{24}$ This is consistent with our finding of increased DCX, MAP2, and TUJ1 expression in the exosome-treated group concurrent with the increase in vessel density. These 

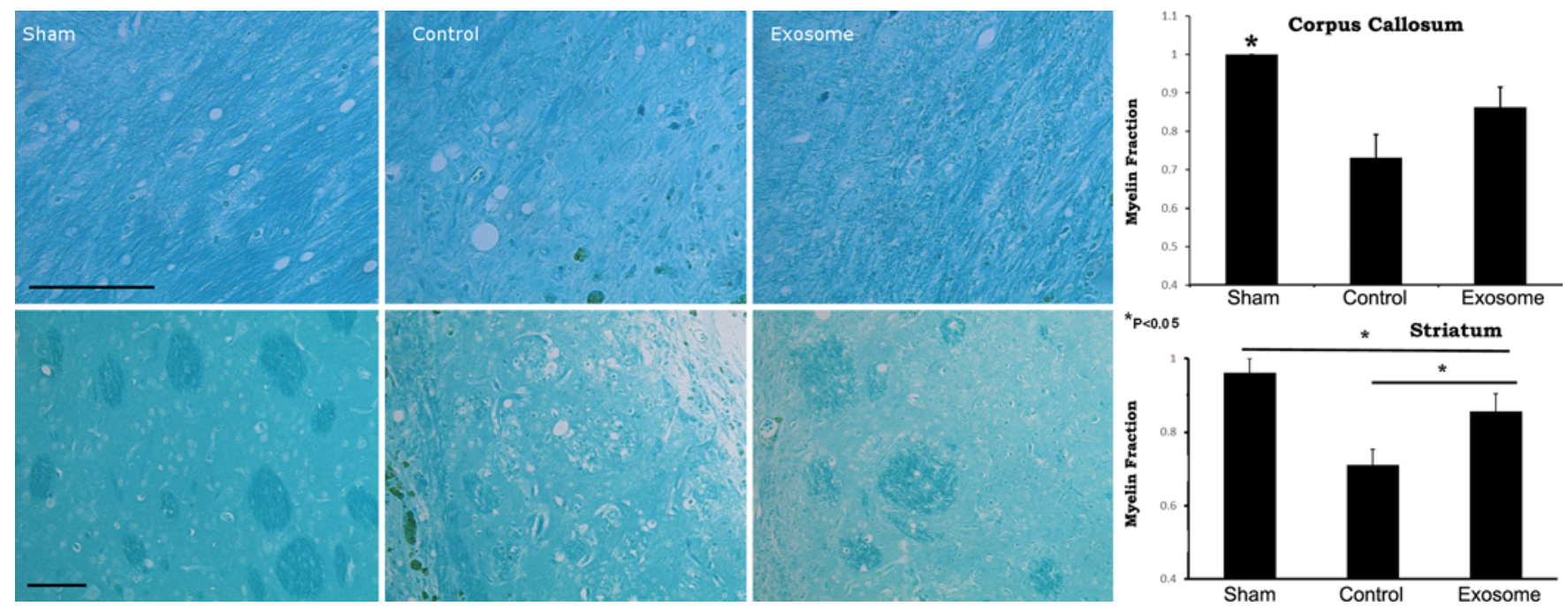

FIG. 7. The myelin-positive area was quantified, then ipsilateral measurements were compared to contralateral measurements. The upper row shows samples from the corpus callosum, the lower row shows samples from the striatum. The control and exosome groups had less myelin in the corpus callosum $(p<0.05)$ than the sham group. In the striatal bundles, the exosome group showed greater recovery of white matter compared to controls $(p<0.05)$. Data in bar graphs represent mean $\pm S E($ error bars); $n=$ 8 per group. Both scale bars $=100 \mu \mathrm{m}$; original magnification $\times 200$ (upper row) and $\times 400$ (lower row).

mechanisms may be responsible for the angiogenic contribution to functional recovery after ICH, as demonstrated by our group and others. $25,33,50,53,55,57$

Newly generated neurons were identified by double labeling for BrdU (Figs. 5 and 6) and 3 neuronal markers. Exosome treatment significantly increased the number of SVZ progenitors with neuronal destiny compared to findings in controls. DCX is observed in the earliest stages of neuronal development and during migration. ${ }^{11,31}$ DCX expression is restricted to the neuronal lineage and multipotential precursors, and is observed in the dentate gyrus of the hippocampus, SVZ, rostral migratory stream, and olfactory bulb. ${ }^{45}$ Depletion of DCX cells of the SVZ showed reduced motor recovery after stroke, indicating the importance of these neural progenitor cells (i.e., neuroblasts) for functional recovery. ${ }^{43}$ TUJ1 is present in newly generated immature post-mitotic neurons and differentiated neurons and in some mitotically active neuronal precursors, ${ }^{44}$ TUJ1-expressing cells also possess more neurites. ${ }^{27}$ MAP2 expression appears to be confined to neurons and reactive astrocytes. ${ }^{13}$ Overall, TUJ1 marks the SVZ cells destined for the neuronal lineage, and MAP2 increases as they mature into functioning neurons. ${ }^{8,26}$ Unlike the control group, SVZ cells of the exosome-treated group coexpressed the markers for these different stages of neural differentiation and BrdU, showing that those cells were generated during the 14 days of BrdU administration and matured by day 28. These 3 markers were chosen to show that newborn neurons survive and move into and aid in the remodeling of the border zone. Concentrated conditioned media from cultured SVZ cells promoted functional recovery in a mouse ischemic stroke model, which suggests that beyond the beneficial effect of new neurons in damaged networks, SVZ cells secrete factors such as VEGF that enhance the remodeling of their local environment. ${ }^{10}$

Interestingly, the control ICH group exhibited less neu- rogenesis than the sham group, but in the exosome-treated group neurogenesis markers recovered to the levels seen in the sham group. Usually after ICH there is an increase in neurogenesis, even without treatment. ${ }^{30} \mathrm{We}$ postulate that the 26 1/2-gauge needle tract might have induced neurogenesis in animals in the sham group. Critically, our BrdU treatment stopped 14 days before euthanization. It is possible that in the control ICH many of the posthemorrhage BrdU cells had undergone apoptosis, consistent with Otero and colleagues' findings that ICH-induced neuro-
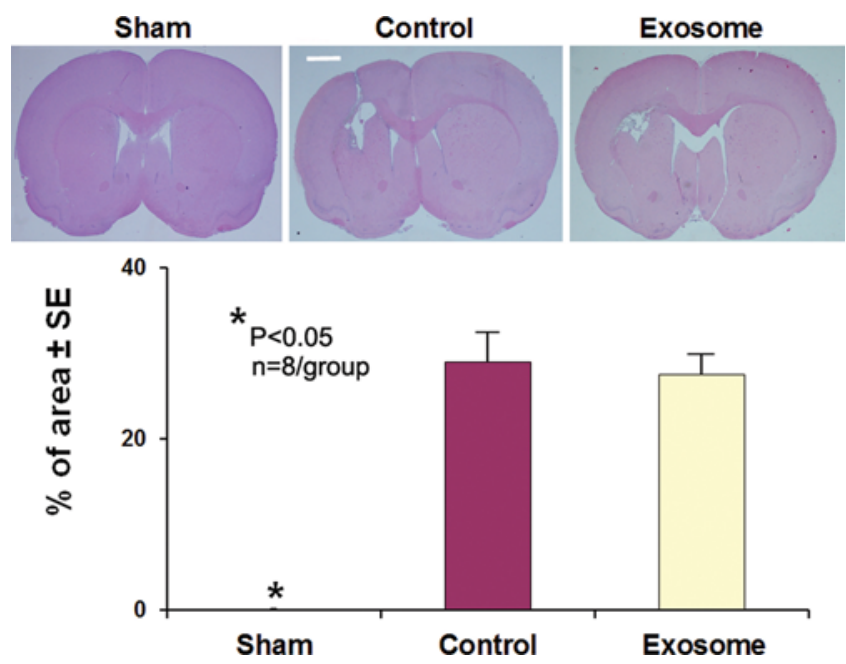

FIG. 8. Exosome treatment did not significantly change striatal tissue loss in the $\mathrm{ICH}$ region relative to the contralateral normal region of rats killed at day 28 after ICH. Upper: Images of $\mathrm{H} \& \mathrm{E}$-stained specimens from the sham, control, and exosome-treated groups (scale bar $=2 \mathrm{~mm}$ ). Lower: Data in the bar graphs represent mean \pm SE (error bars); $n=8$ per group. 
genic neurons survive for only 3 weeks. ${ }^{36}$ This increased apoptosis is consistent with the loss of other neurogenic markers, DCX, TUJ1, and MAP2, in the control animals. Exosome treatment may have increased the endogenous neurogenesis or enhanced the survival of newborn cells, resulting in the observed findings.

In comparison to the control group, the exosome-treated group had increased white matter density in striatal bundles $(\mathrm{p}<0.05)$ around the ICH border zone and, to a lesser degree, increased white matter density in the corpus callosum $(\mathrm{p}=0.12)$. Another time-course experiment using the ICH model showed that at 7 days, when white matter bundles were most fragmented, oligodendrocytic precursors migrated into the bundles and matured into myelinating oligodendrocytes. These white matter bundles were substantially more intact at day 14 than at day $7 .{ }^{20}$ This creates the possibility that the increased white matter observed in our study's exosome-treated group was due to remyelination by newly generated oligodendrocytes, some originating from the SVZ, rather than a protective effect against hemorrhagic damage. ${ }^{54,56}$ Similar to the mutualism between neuron and endothelial proliferation, endothelial cells provide fibroblast growth factor (FGF) to increase oligodendrocyte progenitor cell (OPC) proliferation. OPCs produce MMP9 specifically during white matter injurywhich has the dual effect of increasing BBB permeability and stimulating angiogenesis. ${ }^{18,32}$ Our finding that exosomes increased vessel density and myelin-positive area in the striatum supports this hypothesis of oligodendrocyticendothelial mutualism.

The finding of improved brain remodeling in our study is consistent with similar studies of exosomes in models of TBI and ischemic stroke. Although the etiology (ectopic serum proteins, oxygen deprivation, and physical trauma) of tissue damage is varied, the features of a central core of neuronal death, loss of neurological function, and a surrounding region of temporal and dynamic cellular vulnerability are similar. ${ }^{20,51}$ The gradual recovery of function over the course of a month in our experiment suggests a primary mechanism of brain remodeling over the predominantly neuroprotective mechanism.

Otero-Ortega et al. performed a similar study using an exosome treatment in a collagenase rat model of $\mathrm{ICH} .{ }^{37} \mathrm{In}$ their experiment they used cultured MSCs obtained from allogeneic adipose tissue of Sprague-Dawley rats (250$300 \mathrm{~g}$ ), whereas our MSCs were derived from bone marrow (their exosome preparation kit was miRCURY, ours was ExoQuick).

Their exosome group had increased BDA-labeled axons and MRI fiber tract density compared to control, indicating axonal sprouting. CryoMyelin staining found restored myelin in the exosome group. Markers of OPC differentiation such as CNPase and MOG were also increased in the exosome group. This is consistent with our hypothesis that OPC differentiation may have resulted in the increase in Luxol fast blue staining observed in our rats. However, they found a decrease in lesion size in the treated group compared to controls, whereas we found no change. The therapeutic constituents of their adipocyte-derived exosomes may be different from those of our bone marrowderived exosomes. The collagenase model induces a blood effusion but perhaps not to the same extent as our direct blood infusion.

Some limitations should be noted in the present study. There was lack of an effective way to quantify the number of exosomes reaching the target region by intravenous administration. Other authors have infused exosomes with fluorescently labeled chemotherapies and found that the fluorescence passed beyond the BBB and into the brain tissue in zebrafish. ${ }^{52}$ Endothelial exosomes were better than cancer exosomes at penetrating the BBB, presumably because of receptor proteins such as CD63 expressed on the exosomes and aiding in endocytosis. Adapting a similar labeling method for our exosomes will help verify a doseresponse and location response after experimental $\mathrm{ICH}$.

We cannot exclude the possibility that microvesicle components are in the prepared exosome precipitate even though the supernatant was carefully removed and the pellet was washed after centrifugation. In addition, exosomes from other cell types may have a different effect on $\mathrm{ICH}$ recovery than MSC-derived exosomes and might be employed as more ideal comparison controls besides PBS. In this proof-of-concept study, we focused on gross morphological changes over alterations in protein expression in the recovering tissue. It would be prudent to establish a time course for the exosomes' therapeutic effects by having multiple euthanization points as well as different exosome dosing schedules.

Our study did not identify the specific contents of the exosomes or the transfer of exosome content to recipient cells. However, other studies by our laboratory have used a luciferase reporter system to show that MSCs can transfer miRNAs to other cell types via exosomes. ${ }^{48}$ MSCs enhanced with miRNA 133b increase cortical axonal density after stroke beyond the positive affect of naïve MSCs. ${ }^{51}$ Since exosomes pick up constituents of the cytoplasm, MSCs modified with high-expression miRNA plasmids will generate exosomes abundant in that miRNA. ${ }^{47}$ The variety of endogenous mechanisms that miRNAs support makes miRNA-enhanced exosomes a versatile pharmaceutical delivery system. Exosomes also contain proteins, lipids, and long noncoding RNA that can be transferred with miRNA to recipient cells. ${ }^{12,23}$ Exosomes may act on different cell types in the brain, and we cannot identify from our study whether there is one major target that starts a chain reaction or multiple targets that generally promote neurovascular remodeling, neurogenesis, and functional recovery after $\mathrm{ICH}$.

\section{Conclusions}

We have demonstrated that intravenous administration of MSC-derived exosomes improves functional recovery and promotes neurovascular and white matter remodeling in rats after ICH. The therapeutic benefits observed in this study coincided with increased cell proliferation, vascularity, white matter density, and neurogenesis. This observation opens up a novel avenue for treatment of $\mathrm{ICH}$ and possibly other neurological diseases.

\section{Acknowledgments}

We would like to thank Susan MacPhee-Gray for her help with manuscript editing and formatting. 


\section{References}

1. Brody DL, Holtzman DM: Morris water maze search strategy analysis in PDAPP mice before and after experimental traumatic brain injury. Exp Neurol 197:330-340, 2006

2. Brody DL, Mac Donald C, Kessens CC, Yuede C, Parsadanian M, Spinner M, et al: Electromagnetic controlled cortical impact device for precise, graded experimental traumatic brain injury. J Neurotrauma 24:657-673, 2007

3. Chen J, Li Y, Katakowski M, Chen X, Wang L, Lu D, et al: Intravenous bone marrow stromal cell therapy reduces apoptosis and promotes endogenous cell proliferation after stroke in female rat. J Neurosci Res 73:778-786, 2003

4. Chen J, Zacharek A, Zhang C, Jiang H, Li Y, Roberts C, et al: Endothelial nitric oxide synthase regulates brain-derived neurotrophic factor expression and neurogenesis after stroke in mice. J Neurosci 25:2366-2375, 2005

5. Chieregato A, Tagliaferri F, Tanfani A, Cocciolo F, Benedettini W, Compagnone $\mathrm{C}$, et al: Cerebral blood flow in mean cerebral artery low density areas is not always ischemic in patients with aneurysmal subarachnoid hemorrhage--relationship with neurological outcome. Acta Neurochir Suppl 95:153-158, 2005

6. Chopp M, Li Y, Zhang ZG: Mechanisms underlying improved recovery of neurological function after stroke in the rodent after treatment with neurorestorative cell-based therapies. Stroke 40 (3 Suppl):S143-S145, 2009

7. Chopp M, Zhang ZG: Emerging potential of exosomes and noncoding microRNAs for the treatment of neurological injury/diseases. Expert Opin Emerg Drugs 20:523-526, 2015

8. Dehmelt L, Halpain S: The MAP2/Tau family of microtubule-associated proteins. Genome Biol 6:204, 2005

9. Doeppner TR, Herz J, Görgens A, Schlechter J, Ludwig AK, Radtke S, et al: Extracellular vesicles improve post-stroke neuroregeneration and prevent postischemic immunosuppression. Stem Cells Transl Med 4:1131-1143, 2015

10. Doeppner TR, Traut V, Heidenreich A, Kaltwasser B, Bosche B, Bähr M, et al: Conditioned medium derived from neural progenitor cells induces long-term post-ischemic neuroprotection, sustained neurological recovery, neurogenesis, and angiogenesis. Mol Neurobiol 54:1531-1540, 2017

11. Francis F, Koulakoff A, Boucher D, Chafey P, Schaar B, Vinet $\mathrm{MC}$, et al: Doublecortin is a developmentally regulated, microtubule-associated protein expressed in migrating and differentiating neurons. Neuron 23:247-256, 1999

12. Garnier D, Magnus N, Meehan B, Kislinger T, Rak J: Qualitative changes in the proteome of extracellular vesicles accompanying cancer cell transition to mesenchymal state. Exp Cell Res 319:2747-2757, 2013

13. Geisert EE Jr, Johnson HG, Binder LI: Expression of microtubule-associated protein 2 by reactive astrocytes. Proc Natl Acad Sci U S A 87:3967-3971, 1990

14. Gu Y, Gong Y, Liu WQ, Keep RF, Xi G, Hua Y: Zinc protoporphyrin attenuates white matter injury after intracerebral hemorrhage. Acta Neurochir Suppl 121:199-202, 2016

15. Hemphill JC III, Greenberg SM, Anderson CS, Becker K, Bendok BR, Cushman M, et al: Guidelines for the management of spontaneous intracerebral hemorrhage: a guideline for healthcare professionals from the American Heart Association/ American Stroke Association. Stroke 46:2032-2060, 2015

16. Huang J, Kodithuwakku ND, He W, Zhou Y, Fan W, Fang W, et al: The neuroprotective effect of a novel agent $\mathrm{N} 2$ on rat cerebral ischemia associated with the activation of PI3K/Akt signaling pathway. Neuropharmacology 95:12-21, 2015

17. Huang L, Ma W, Ma Y, Feng D, Chen H, Cai B: Exosomes in mesenchymal stem cells, a new therapeutic strategy for cardiovascular diseases? Int J Biol Sci 11:238-245, 2015

18. Itoh K, Maki T, Lok J, Arai K: Mechanisms of cell-cell interaction in oligodendrogenesis and remyelination after stroke. Brain Res 1623:135-149, 2015
19. Janas T, Janas MM, Sapoń K, Janas T: Mechanisms of RNA loading into exosomes. FEBS Lett 589:1391-1398, 2015

20. Joseph MJ, Caliaperumal J, Schlichter LC: After intracerebral hemorrhage, oligodendrocyte precursors proliferate and differentiate inside white-matter tracts in the rat striatum. Transl Stroke Res 7:192-208, 2016

21. Kempermann G, Gage FH: Neurogenesis in the adult hippocampus. Novartis Found Symp 231:220-241, 302-306, 2000

22. Kojima T, Hirota Y, Ema M, Takahashi S, Miyoshi I, Okano $\mathrm{H}$, et al: Subventricular zone-derived neural progenitor cells migrate along a blood vessel scaffold toward the post-stroke striatum. Stem Cells 28:545-554, 2010

23. Lang HL, Hu GW, Chen Y, Liu Y, Tu W, Lu YM, et al: Glioma cells promote angiogenesis through the release of exosomes containing long non-coding RNA POU3F3. Eur Rev Med Pharmacol Sci 21:959-972, 2017

24. Leventhal C, Rafii S, Rafii D, Shahar A, Goldman SA: Endothelial trophic support of neuronal production and recruitment from the adult mammalian subependyma. Mol Cell Neurosci 13:450-464, 1999

25. Liu XS, Zhang ZG, Zhang RL, Gregg S, Morris DC, Wang $\mathrm{Y}$, et al: Stroke induces gene profile changes associated with neurogenesis and angiogenesis in adult subventricular zone progenitor cells. J Cereb Blood Flow Metab 27:564-574, 2007

26. Lledo PM, Alonso M, Grubb MS: Adult neurogenesis and functional plasticity in neuronal circuits. Nat Rev Neurosci 7:179-193, 2006

27. Low WC, Yau WW, Stanton LW, Marcy G, Goh E, Chew SY: Directing neuronal differentiation of primary neural progenitor cells by gene knockdown approach. DNA Cell Biol 31:1148-1160, 2012

28. Lu M, Chen J, Lu D, Yi L, Mahmood A, Chopp M: Global test statistics for treatment effect of stroke and traumatic brain injury in rats with administration of bone marrow stromal cells. J Neurosci Methods 128:183-190, 2003

29. Lucke-Wold BP, Logsdon AF, Manoranjan B, Turner RC, McConnell E, Vates GE, et al: Aneurysmal subarachnoid hemorrhage and neuroinflammation: a comprehensive review. Int J Mol Sci 17:497, 2016

30. Masuda T, Isobe Y, Aihara N, Furuyama F, Misumi S, Kim $\mathrm{TS}$, et al: Increase in neurogenesis and neuroblast migration after a small intracerebral hemorrhage in rats. Neurosci Lett 425:114-119, 2007

31. Maucksch C, McGregor AL, Yang M, Gordon RJ, Yang M, Connor B: IGF-I redirects doublecortin-positive cell migration in the normal adult rat brain. Neuroscience 241:106115,2013

32. Miyamoto N, Pham LD, Seo JH, Kim KW, Lo EH, Arai K: Crosstalk between cerebral endothelium and oligodendrocyte. Cell Mol Life Sci 71:1055-1066, 2014

33. Morris DC, Zhang ZG, Zhang J, Xiong Y, Zhang L, Chopp M: Treatment of neurological injury with thymosin $\beta 4$. Ann N Y Acad Sci 1269:110-116, 2012

34. Nguyen AP, Huynh HD, Sjovold SB, Colbourne F: Progressive brain damage and alterations in dendritic arborization after collagenase-induced intracerebral hemorrhage in rats. Curr Neurovasc Res 5:171-177, 2008

35. O'Dell SJ, Feinberg LM, Marshall JF: A neurotoxic regimen of methamphetamine impairs novelty recognition as measured by a social odor-based task. Behav Brain Res 216:396-401, 2011

36. Otero L, Zurita M, Bonilla C, Rico MA, Aguayo C, Rodriguez A, et al: Endogenous neurogenesis after intracerebral hemorrhage. Histol Histopathol 27:303-315, 2012

37. Otero-Ortega L, Gómez de Frutos MC, Laso-García F, Rodríguez-Frutos B, Medina-Gutiérrez E, López JA, et al: Exosomes promote restoration after an experimental animal 
model of intracerebral hemorrhage. J Cereb Blood Flow Metab [epub ahead of print], 2017

38. Pelz J, Härtig W, Weise C, Hobohm C, Schneider D, Krueger $\mathrm{M}$, et al: Endothelial barrier antigen-immunoreactivity is conversely associated with blood-brain barrier dysfunction after embolic stroke in rats. Eur J Histochem 57:e38, 2013

39. Seyfried D, Ding J, Han Y, Li Y, Chen J, Chopp M: Effects of intravenous administration of human bone marrow stromal cells after intracerebral hemorrhage in rats. J Neurosurg 104:313-318, 2006

40. Seyfried DM, Han Y, Yang D, Ding J, Chopp M: Erythropoietin promotes neurological recovery after intracerebral haemorrhage in rats. Int J Stroke 4:250-256, 2009

41. Seyfried DM, Han Y, Yang D, Ding J, Savant-Bhonsale S, Shukairy MS, et al: Mannitol enhances delivery of marrow stromal cells to the brain after experimental intracerebral hemorrhage. Brain Res 1224:12-19, 2008

42. Seyfried DM, Han Y, Yang D, Ding J, Shen LH, SavantBhonsale S, et al: Localization of bone marrow stromal cells to the injury site after intracerebral hemorrhage in rats. $\mathbf{J}$ Neurosurg 112:329-335, 2010

43. Sun F, Wang X, Mao X, Xie L, Jin K: Ablation of neurogenesis attenuates recovery of motor function after focal cerebral ischemia in middle-aged mice. PLoS One 7:e46326, 2012

44. von Bohlen Und Halbach O: Immunohistological markers for staging neurogenesis in adult hippocampus. Cell Tissue Res 329:409-420, 2007

45. Walker TL, Yasuda T, Adams DJ, Bartlett PF: The doublecortin-expressing population in the developing and adult brain contains multipotential precursors in addition to neuronal-lineage cells. J Neurosci 27:3734-3742, 2007

46. Wei Y, Yemisci M, Kim HH, Yung LM, Shin HK, Hwang SK, et al: Fingolimod provides long-term protection in rodent models of cerebral ischemia. Ann Neurol 69:119-129, 2011

47. Xin H, Katakowski M, Wang F, Qian JY, Liu XS, Ali MM, et al: MicroRNA cluster miR-17-92 cluster in exosomes enhance neuroplasticity and functional recovery after stroke in rats. Stroke 48:747-753, 2017

48. Xin H, Li Y, Buller B, Katakowski M, Zhang Y, Wang X, et al: Exosome-mediated transfer of miR-133b from multipotent mesenchymal stromal cells to neural cells contributes to neurite outgrowth. Stem Cells 30:1556-1564, 2012

49. Xin H, Li Y, Chopp M: Exosomes/miRNAs as mediating cell-based therapy of stroke. Front Cell Neurosci 8:377, 2014

50. Xin H, Li Y, Cui Y, Yang JJ, Zhang ZG, Chopp M: Systemic administration of exosomes released from mesenchymal stromal cells promote functional recovery and neurovascular plasticity after stroke in rats. J Cereb Blood Flow Metab 33:1711-1715, 2013
51. Xin H, Li Y, Liu Z, Wang X, Shang X, Cui Y, et al: MiR$133 \mathrm{~b}$ promotes neural plasticity and functional recovery after treatment of stroke with multipotent mesenchymal stromal cells in rats via transfer of exosome-enriched extracellular particles. Stem Cells 31:2737-2746, 2013

52. Yang T, Martin P, Fogarty B, Brown A, Schurman K, Phipps $\mathrm{R}$, et al: Exosome delivered anticancer drugs across the blood-brain barrier for brain cancer therapy in Danio rerio. Pharm Res 32:2003-2014, 2015

53. Zacharek A, Chen J, Cui X, Li A, Li Y, Roberts C, et al: Angiopoietin1/Tie 2 and VEGF/Flk1 induced by MSC treatment amplifies angiogenesis and vascular stabilization after stroke. J Cereb Blood Flow Metab 27:1684-1691, 2007

54. Zhang R, Chopp M, Zhang ZG: Oligodendrogenesis after cerebral ischemia. Front Cell Neurosci 7:201, 2013

55. Zhang Y, Chopp M, Meng Y, Katakowski M, Xin H, Mahmood A, et al: Effect of exosomes derived from multipluripotent mesenchymal stromal cells on functional recovery and neurovascular plasticity in rats after traumatic brain injury. $\mathbf{J}$ Neurosurg 122:856-867, 2015

56. Zhang Z, Chopp M: Neural stem cells and ischemic brain. J Stroke 18:267-272, 2016

57. Zhang Z, Yang J, Yan W, Li Y, Shen Z, Asahara T: Pretreatment of cardiac stem cells with exosomes derived from mesenchymal stem cells enhances myocardial repair. J Am Heart Assoc 5:e02856, 2016

58. Zheng H, Chen C, Zhang J, Hu Z: Mechanism and therapy of brain edema after intracerebral hemorrhage. Cerebrovasc Dis 42:155-169, 2016

\section{Disclosures}

The authors report no conflict of interest concerning the materials or methods used in this study or the findings specified in this paper.

\section{Author Contributions}

Conception and design: Chopp. Acquisition of data: Donald Seyfried, Han, Don Seyfried, Meng, Yang. Analysis and interpretation of data: Donald Seyfried, Han, Don Seyfried, Meng, Yang, Schultz. Drafting the article: Donald Seyfried, Don Seyfried. Critically revising the article: Donald Seyfried, Don Seyfried, Chopp. Reviewed submitted version of manuscript: all authors. Statistical analysis: Schultz. Study supervision: Chopp.

\section{Correspondence}

Donald Seyfried: Henry Ford Hospital, Detroit, MI. dseyfri1@ hfhs.org. 